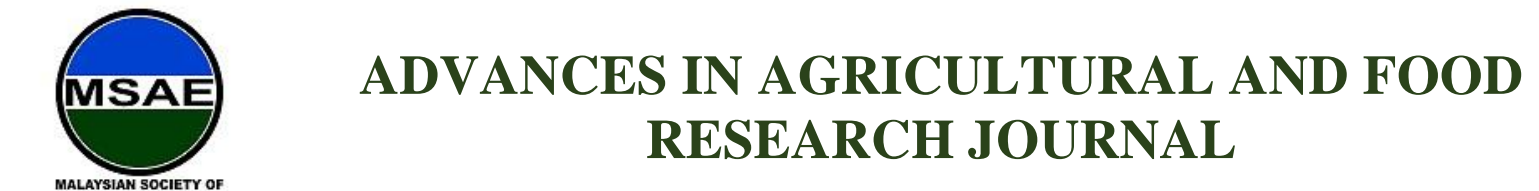

Original Research Article

\title{
Efficacy of Deep Learning Algorithm in Classifying Chilli Plant Growth Stages
}

\begin{abstract}
Danial Mirza Muammar Rozilan ${ }^{1 *}$, Marsyita Hanafi ${ }^{1 *}$, Roslizah Ali ${ }^{1}$, Mohd Adib Razak ${ }^{1}$, Cui Hairu ${ }^{1}$
${ }^{1}$ Department of Computer and Communication System Engineering, Faculty of Engineering, Universiti Putra Malaysia, Serdang 43400, Selangor, Malaysia.

*Corresponding author: Danial Mirza Muammar Rozilan and Marsyita Hanafi, Department of Computer and Communication System Engineering, Faculty of Engineering, Universiti Putra Malaysia, Serdang 43400, Selangor, Malaysia; danialmirza9296@gmail.com and marsyita@upm.edu.my
\end{abstract}

\begin{abstract}
Automatic plant growth monitoring has received considerable attention in recent years. The demand in this field has created various opportunities, especially for automatic classification using deep learning methods. In this paper, the efficiency of deep learning algorithms in classifying the growth stage of chili plants is studied. Chili is one of the high cash value crops, and automatic identification of chili plant growth stages is essential for crop productivity. Nevertheless, the study on automatic chili plant growth stage classification using deep learning approaches is not widely explored, and this is due to the unavailability of public datasets on the chili plant growth stages. Various deep learning methods, namely Inception V3, ResNet50, and VGG16, were used in the study, and the results have shown that these methods performed well in terms of accuracy and stability when tested on a dataset that consists of 2,320 images of Capsicum annum 'Bird's Eye' plants of various growth stages and imaging conditions. Nevertheless, the results have also shown that the deep learning methods have difficulty classifying images with a complex background where more than one chili plant was captured in an image.
\end{abstract}

Keywords: classification; plant growth stages; deep learning

Received: $13^{\text {th }}$ August 2021

Citation: Rozilan, D. M. M., Hanafi,

Received in revised form:

Accepted: $16^{\text {th }}$ September 2021

Available Online: $23^{\text {rd }}$ September 2021 M., Ali, R., et al. (2021). Efficacy of deep learning algorithm in classifying chilli plant growth stages. Adv Agri Food Res J 2021; 2(2):a0000238. https://doi.org/10.36877/aafrj.a0000238

\section{Introduction}

Chili or scientifically known as Capsicum annum is one of high cash crops values in Malaysia (Yusuf et al., 2016), and it has been recognized as among the top tenth of selfsufficiency ratio (SSR) with the highest import dependency ratio (IDR) of $73.6 \%$ 
(Department of Statistics Malaysia, 2020). Capsicum annum 'Bird's Eye' and Capsicum annum 'Red' are among Malaysia's widely domesticated planted chili (Norfadzilah, 2018). Chili plants grow upright with green stem branches, and the leaves have a variety of colors, from light green to dark green (Suhaimi et al., 2016). According to Haifa Group (2021), the growth stage of chili plants can be classified into four stages; the first stage is the stage of vegetative growth that is from planting or seeding to the first flowering. The second stage is the period from flowering to fruit set, and the third stage is from fruit ripening to the first harvest. The last stage is the period from the first harvest to the last harvest. The duration of each stage may vary according to the growing method, characteristics of chili variety, and climatic conditions (Haifa Group, 2021). The growth stage of chili plants is typically measured manually, as discussed by Sharma \& Kumar (2017). The manual way of identifying growth stages is inconsistent because the human factor and the process are time-consuming (Kim et al., 2013). Nevertheless, the manual process still needs to be performed due to the requirement of different amounts of fertilizer and water for each growth stage, which is necessary for optimizing crop yields (Wayne, 2021).

In recent years, the automatic classification of the growth stage of plants using computer vision technology has received significant attention. This is because automatic plant growth classification or prediction is essential for analyzing growth patterns to aid the acceleration of plant cycles, the prediction of phenotypic traits, and the efficient execution of experiments (Yasrab et al., 2021). The classical computer vision approaches are based on conventional image segmentation or structural analysis. The aim is to find the region of interest or structure analysis based on lines, curves, pixel intensities, and differentials (Spalding \& Miller, 2013). The examples of the classical approaches are optimal thresholding and probability distribution methods (Otsu, 1979; Bouman \& Shapiro, 1994) and machine learning approaches (Othman et al., 2010; Yu et al., 2011). The plant growth prediction using machine learning approaches has received significant attention in macroscopic phenotypes studies (Li et al., 2020; Keller et al., 2018; Mochida et al., 2018; Van Dijk et al., 2021). Even so, the problem of achieving high accuracy is still the main issue. This is mainly due to complex backgrounds, various illumination effects, and image sizes (Anugraheni et al., 2019).

Furthermore, the best approach to extract distinguishable plant features that can produce high classification accuracy remains unestablished (Hao et al., 2020). The same situation for the study of chili plant growth classification using machine learning. For 
example, Suroso et al. (2016) showed that artificial neural networks and image processing methods could predict the required amount of water and fertilizer based on the plant image parameters. However, the result was only based on three stages of growth, and the used images were captured under a controlled environment (Soethe et al., 2016).

Advance in data acquisition technology has created enormous opportunities to develop highly accurate and efficient classifiers such as deep learning classifiers. Deep learning refers to artificial neural network architectures with a significantly large number of layers of processing that can be easily adapted to changing environments (Learning \& Rai, 2021). There are four categories of deep learning methods, namely Convolutional Neural Networks (CNNs), Restricted Boltzmann Machines (RBMs), Autoencoder, and Sparse Coding (Guo et al., 2016). Among all the methods, Convolutional Neural Networks (CNNs) have been popularly used. A CNN consists of three main neural layers: convolutional layers, pooling layers, and fully connected layers. As discussed by Guo et al. (2016), there are many variations of the $\mathrm{CNN}$ methods, where the difference is basically in terms of the total number of convolutional layers. The CNN classifiers have demonstrated exceptional accuracy and precision in many agriculture applications (Khanramaki et al., 2021; Alhnaity et al., 2020; Ayan et al., 2020; Chouhan et al., 2019; Ferentinos, 2018; Habiba et al., 2019), including in the field of plant phenotyping (Fuentes et al., 2019; Wang et al., 2019; Subetha et al., 2021).

Plant phenotyping is defined as assessing complex plant traits growth, resistance, architecture, physiology, and ecology by Li et al. (2015). Several works on plant phenotypes prediction based on spatial and temporal features of plant growth have been reported (Namin et al., 2018; Sakurai et al., 2019). Namin et al. (2018) reported that a combination of a multimodel Convolutional Neural Network with Long Short-Term Memory (LSTM) approaches offers exceptional accuracy for plant phenotypes and genotypes prediction, which is helpful in the automation of plant production and care. Sakurai et al. (2019) studied the performance of LSTMs with an encoder-decoder model for predicting the growth of plant leaves, where they found that this approach has limited capabilities for several data sets. Nevertheless, the study on the prediction of chili growth stages using deep learning has yet to be explored. The studies related to chili plants classify nutrient deficiency and quality (Bahtiar et al., 2020; Sudianto et al., 2020). Moreover, automatic classification of chili plants' growth stage using the images captured with a complex background and under various imaging conditions such as different scales, various illuminations, and angles has not yet been studied. Inspired by this fact, the efficiency of high-performance $\mathrm{CNN}$ algorithms, which are EfficientNet, 
VGG16, ResNet50, and InceptionV3, in classifying the stages of chili plant growth from the images with various imaging conditions is studied

This paper is organized as follows. Section 2 describes the dataset used in the experiment that consists of chili growth images under an uncontrolled environment, followed by the explanations on VGG, ResNet50, InceptionV3, and EfficientNet architectures in Section 3. Section 4 presents the result and discussion of this study and conclusions in Section 5.

\section{Materials and Methods}

\subsection{Chilli Plant Growth Dataset}

This study's chili plant growth dataset consists of 2,320 images of Capsicum annum 'Bird's Eye' plants. The images were captured at all growth stages, ranging from seedling to harvesting stages, as described in Table 1. The images were collected every week under an uncontrolled environment and various image acquisition parameters, as in Table 2, to reflect the real-life scenarios. These images were captured in an open space in Kuantan, Malaysia, using the Samsung Galaxy S10 Plus. The phone consists of triple cameras with a 12megapixel $12 \mathrm{~mm}$ wide-angle rear lens, a 12-megapixel telephoto lens, and a 16-megapixel ultrawide lens. The dimension of the produced images is 3024 x 4032 pixels. The parameters considered during image acquisition to create challenges for the classifiers are summarized in Table 2. These parameters are based on the situations when capturing the images in reallife scenarios.

Table 1. Chili growth stages (Majlis Ilmu, Kerajaan Negara Brunei Darussalam, 2018)

\begin{tabular}{cc}
\hline Growth Stage & Days \\
Seedling and transplanting & $0-30$ \\
Vegetative & $31-62$ \\
Flowering & $57-92$ \\
Fruiting & $88-120$ \\
\hline
\end{tabular}

Table 2. Summary of the image acquisition parameters.

\begin{tabular}{ll}
\hline \multicolumn{1}{c}{ Parameters } & \multicolumn{1}{c}{ Description } \\
\hline Various distances & The distances of the camera facing the leaf range from $30 \mathrm{~cm}$ to $50 \mathrm{~cm}$. \\
Various views & Three types of views were considered, namely top, side, and front views. \\
Various illuminations & The images were captured in the morning (8 AM to 9 AM) and afternoon \\
& $(12 \mathrm{PM}$ to $1 \mathrm{PM})$. \\
\hline
\end{tabular}



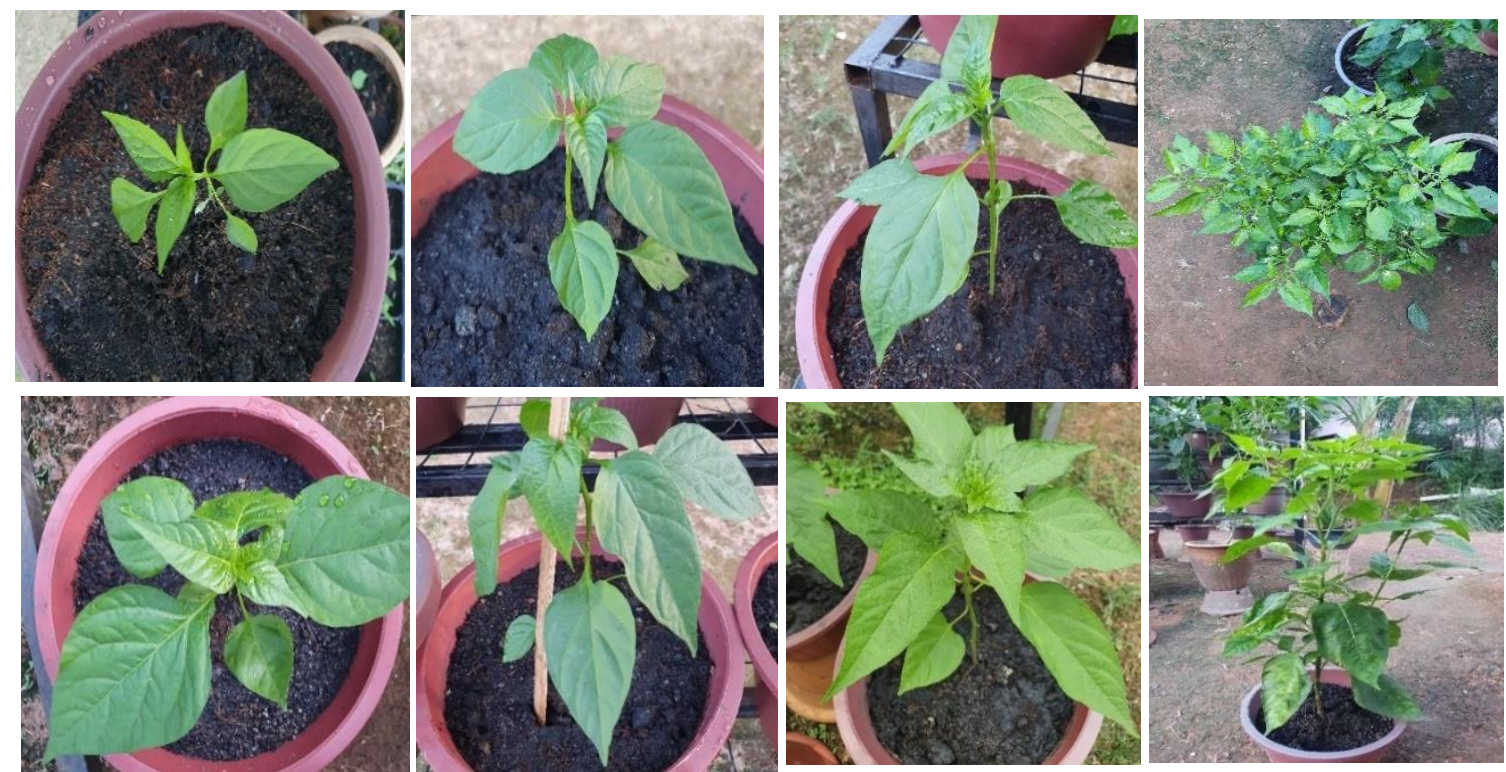

Figure 1. Some examples of the chili plant images used in the experiment.

Chili plant images under extreme exposure, namely underexposed and overexposed, with a complex background, were also considered. The underexposed and overexposed effects are due to the various illumination effects in the morning and afternoon, which are influenced by various weather conditions during the 22 weeks of the data collection. Image acquisition settings were not changed during image acquisition. These images were captured from the chili plants in a rain shelter at the Faculty of Engineering, Universiti Putra Malaysia, as shown in Figure 2. The total number of images is 480 .

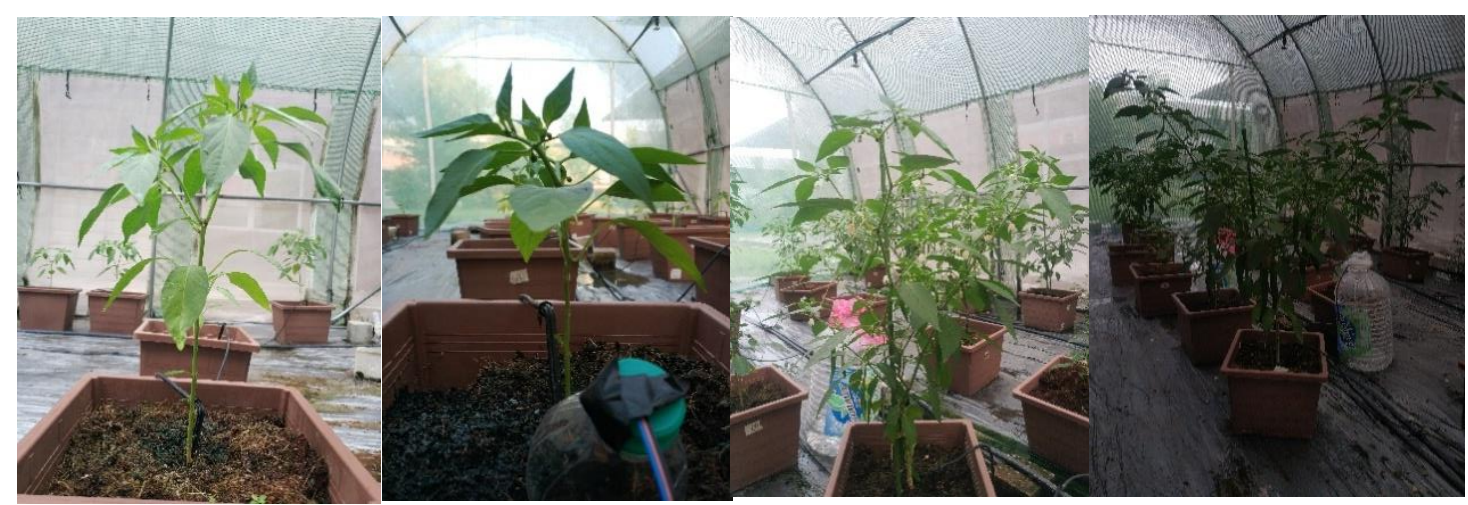

Figure 2. Some examples of images under extreme exposure.

\subsection{Experimental Setup}

In the experiments, the performance of the selected deep learning algorithms in classifying the chili plant growth stages was compared. All the algorithms were trained and 
tested using the images from the developed dataset with a distribution of $80 \%$ and $20 \%$, respectively. Hence, the total number of images used for training and testing for the images collected in Kuantan are 1,952 images and 488 images, respectively. The classification of the growth stage of chili plants is based on the weekly growth stages, which is based on the weekly data collection. As the data were collected for 22 weeks, 22 classes were used in the experiments, ranging from seeding to harvest.

The experiments were executed using Python in a Jupyter notebook environment, running on Intel ${ }^{\circledR}$ Core $^{\mathrm{TM}}$ i5-5200U processor with a Turbo Core Technology Up to $2.7 \mathrm{GHz}$ and 8GB DDR3L RAM. Adam optimizer was also used to reduce loss due to inappropriate selection of learning rate and weights in the algorithm. According to Kingma et al. (2014), this optimizer is computationally efficient and well suited for significant problems in terms of data or parameters. The images used for training and testing were resized to $128 \times 128$ pixels, and these images were selected randomly by python software. The selection of the input image size is based on the recommendation by Habiba et al. (2020) and Sujatha et al. (2021). The small image size should be used for optimizing classifier performance. Other than that, the batch size, which is the number of samples to be processed, is set to 32, and the total number of epochs that is the total number of iterations, is set to 10 .

\subsection{Deep Learning Algorithm Architectures}

According to Chouhan et al. (2019), deep learning is a subset of machine learning. The architecture consists of many processing layers and optimizers that are suitable for efficiently classifying complex problems (Sujatha et al., 2021; Ferentinos, 2018). Alhnaity et al. (2020) claimed that the complex models employed in deep learning could improve classification accuracy and reduce regression problems. In this study, the efficiency of four widely used deep learning algorithms, namely VGG16, ResNet50, Inception V3, and EffcientNet algorithms, in classifying the growth stage of chili plants is compared. The architectures of the selected deep learning algorithms are explained in the following subsections.

\subsubsection{VGG16}

Simonyan and Zisserman developed visual Geometry Group 16 or VGG16 (2015). This algorithm achieved $71.3 \%$ in the top five accuracy category and $90.1 \%$ in the top one accuracy when tested using the ImageNet dataset. The algorithm has shown good performance when classifying leaves or plants (Habiba et al., 2019; Rangarajan \& 
Purushothaman, 2020). The algorithm consists of 16 layers that combine 13 convolutional layers, five top pooling layers between the convolutional layers, and three fully connected layers (FC). In the convolutional layers, various feature maps are generated through the convolution of images using various kernels. The pooling layer is used to reduce the dimensions of feature maps and network parameters. A rectified linear activation function (ReLU) in the architecture is applied to the first two FC layers. This is because the function allows faster learning and decreases the likelihood of vanishing gradient problems. At the final FC layer, a softmax function is used to normalize the classification vector. The VGG model processes the input image and outputs as a vector of thousands of values. The vector represents the probability of classification for the corresponding class. Figure 3 shows the architecture of VGG16.

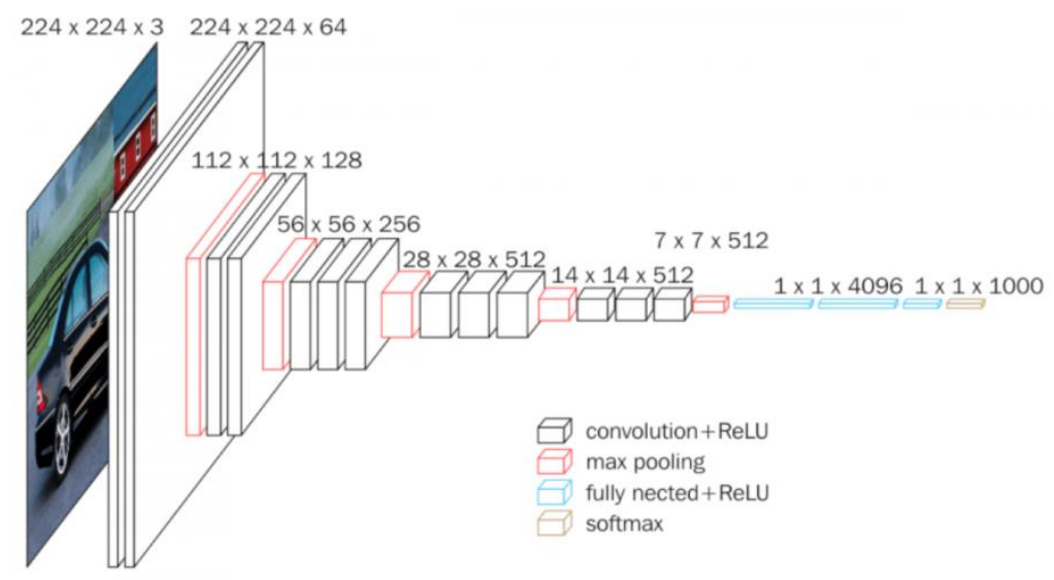

Figure 3. VGG16 Architecture (Zhang et al., 2016)

\subsubsection{ResNet50}

ResNet or Residual Network was introduced in 2015 by He et al. (2016), and the algorithm has shown exceptional performance when classifying the ImageNet dataset. The algorithm consists of a combination of 50 layers deep of convolutional block and identity block, in which each block has three convolutional layers. ResNet utilizes the skip connection concept that allows the algorithm to learn an identity function that would enable the higher layer to perform well as the lower layer. Figure 4 shows the architecture of ResNet50. 


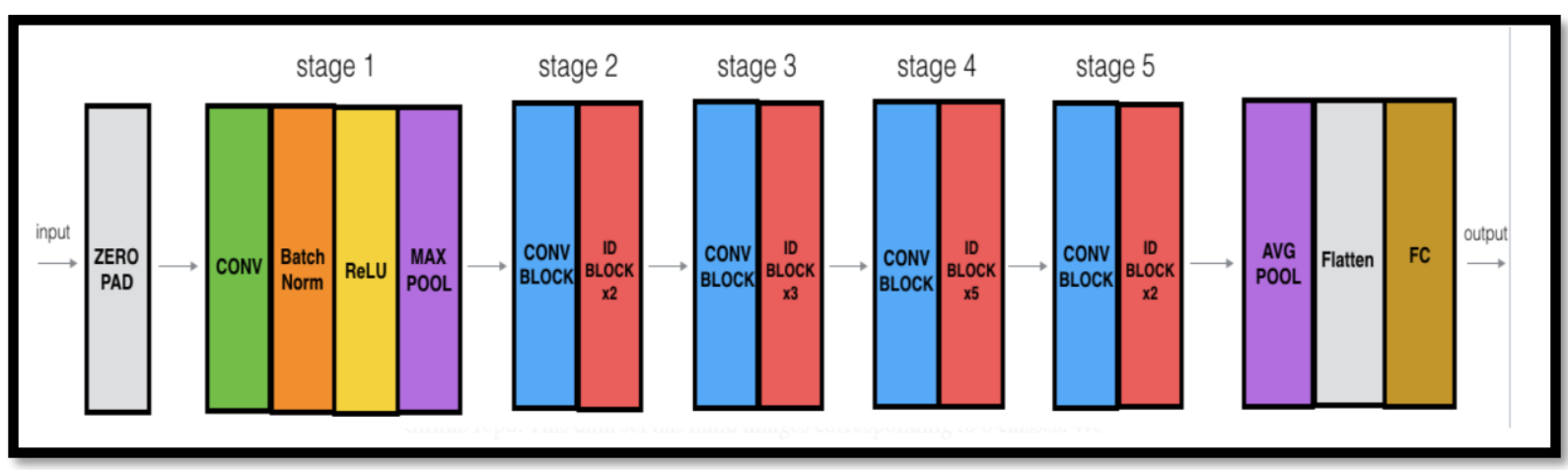

Figure 4. ResNet50 Architecture (Peng et al., 2019)

\subsubsection{Inception V3}

Inception $\mathrm{V} 3$ is a convolutional neural network $(\mathrm{CNN})$ architecture from the Inception model that consists of 48 layers (Sujatha et al., 2021). The algorithm has some advanced features, such as factorized convolutions, and it can lower the number of computational complexities because of the efficient grid size reduction (Ayan et al., 2020). Inception architecture uses an image model block to approximate an optimal local sparse structure in a CNN that allows for more effective computation. The applied dimensionality reduction has made the algorithm more efficient. The Inception V3 has also proven to significantly reduce the number of parameters and computational complexity with efficient grid size reduction. Figure 5 shows the architecture of Inception V3.

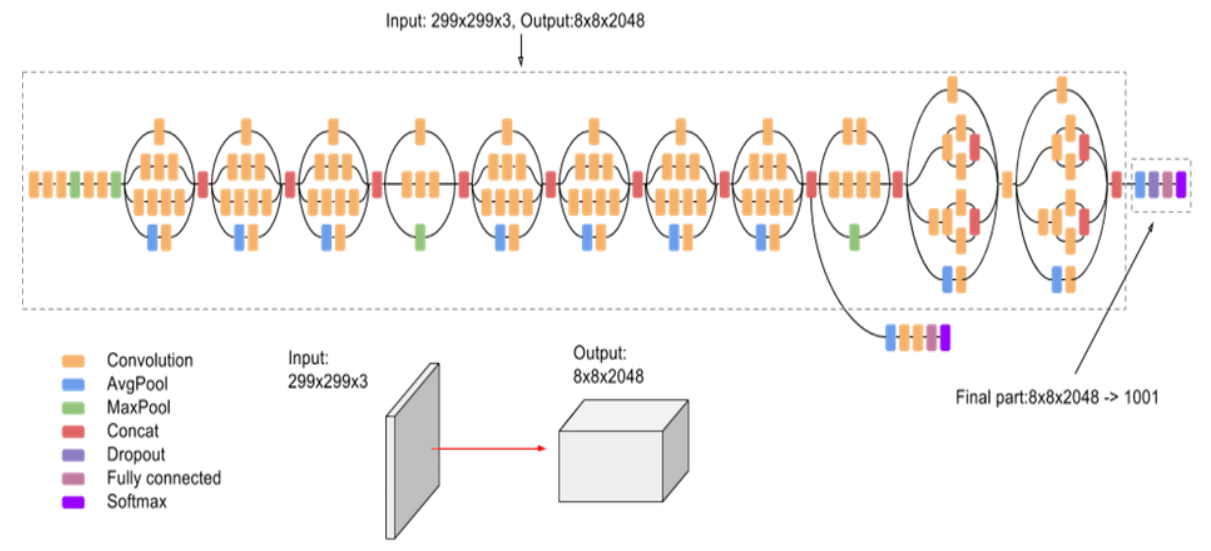

Figure 5. Inception V3 Architecture (Szegedy et al., 2015)

\subsubsection{EfficientNet}

EffcientNet was developed based on model scaling, in which the algorithm uses compound scaling to rescale depth, width, and image resolution. The algorithm has seven versions, and all the versions utilize Inverted Residual Blocks or MBConv Block, where a 
single convolutional filter is applied to each input channel for better efficiency (Tan \& Le, 2019; Sandler et al., 2018). In this experiment, only the first three versions of EfficientNet algorithms were considered: EfficientNet B0, EfficientNet B1, and EfficientNet B2. Figure 6 shows the basic version, which is EfficientNet B0. Each version is upgraded in terms of increasing the number of Module 3 and different repetition patterns. According to Tan \& Le (2019), EfficientNet algorithms have also shown good performance when classifying images from ImageNet. Inspired by this finding, the performance of the EfficientNet algorithm in classifying chili plant growth stages under uncontrolled conditions is investigated. This is to see how well the baseline and the advanced architectures classify complex images.

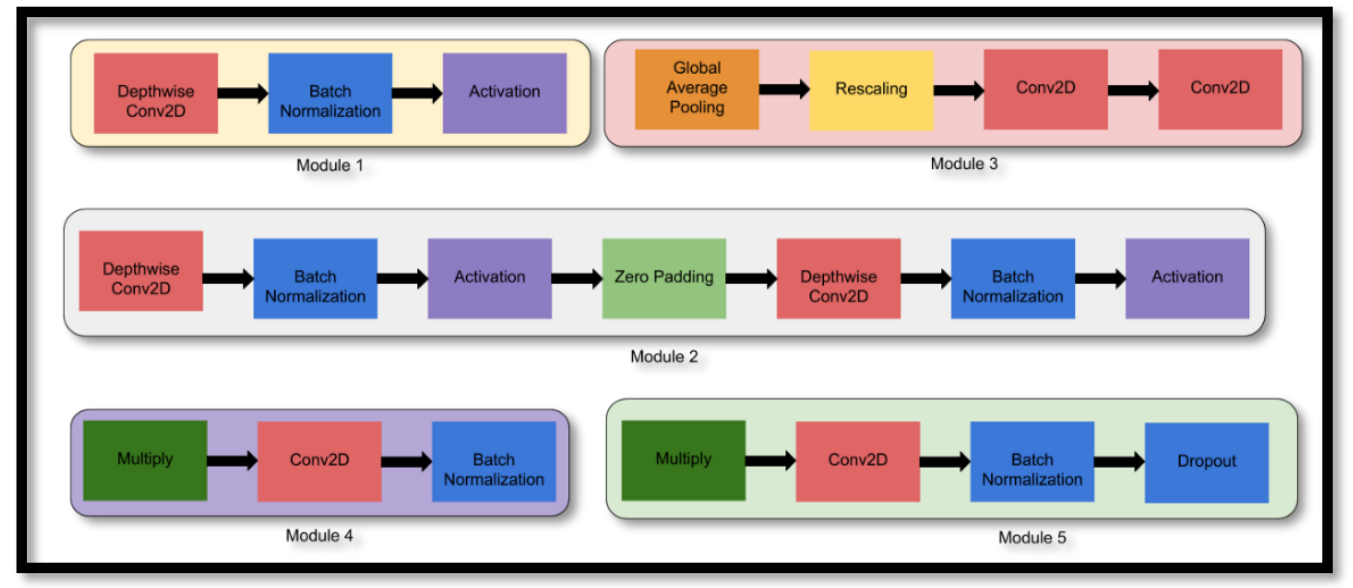

Figure 6. Efficient Net B0 architecture.

\section{Results and Discussions}

The performance of the selected deep learning algorithms was evaluated based on the accuracy and loss, as in equation (1) and equation (2). The accuracy is the number of correctly identified samples during the cross-entropy loss between actual and predicted data.

$$
\begin{aligned}
& \text { Accuracy }=\frac{T P+T N}{T P+T N+F P+F N} \\
& \text { Loss }=\sum_{j=1}^{K} n_{j} \log \left(P_{j}\right)
\end{aligned}
$$

TP, TN, FP, and FN are genuinely positive, true negative, false positive, and false negative, respectively. The training and validation accuracy and loss produced by the VGG16, ResNet50, InceptionV3, and EfficientNet algorithms are illustrated in Figure 7 until Figure 10. 


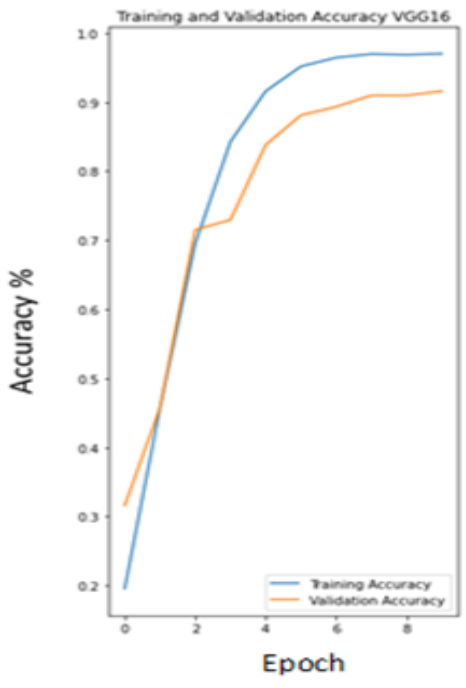

(a)

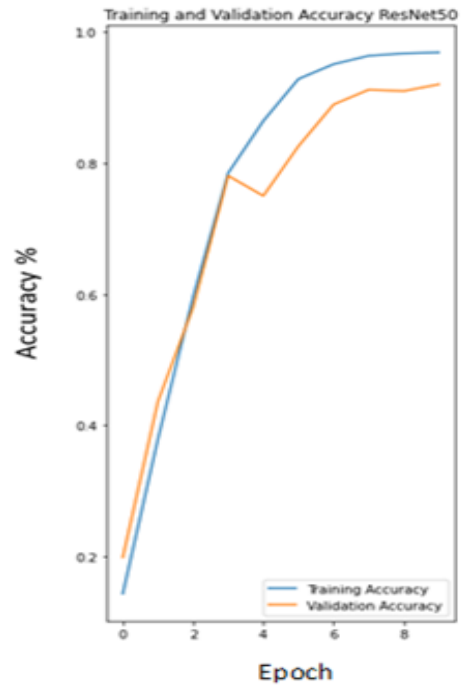

(b)

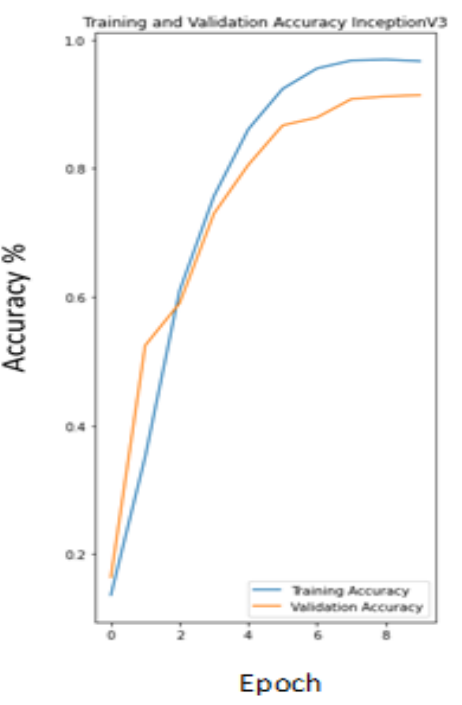

(c)

Figure 7. Training and validation accuracies produced by (a) VGG16, (b) ResNet50, and (c) Inception V3.

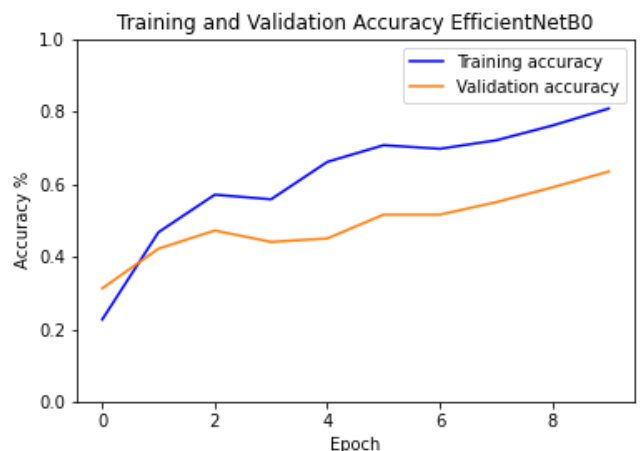

(a)

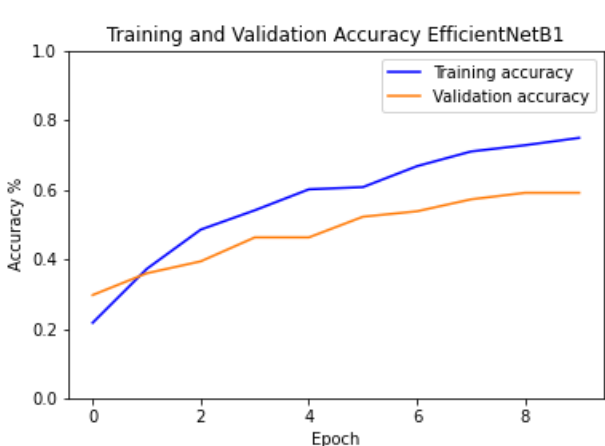

(b)

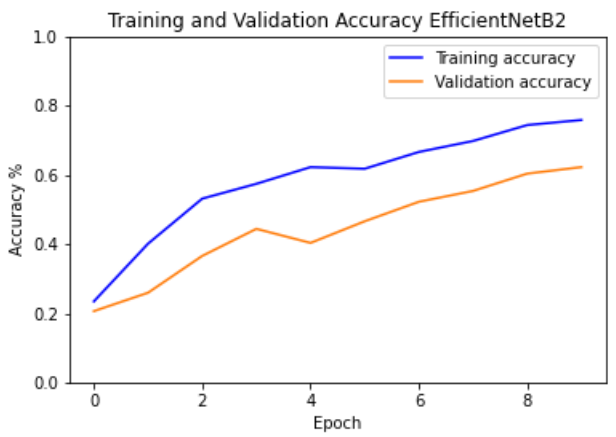

(c)

Figure 8. Training and validation accuracies produced by (a) EfficientNetB0, (b) EfficientNetB1 and (c) EfficientNetB3. 


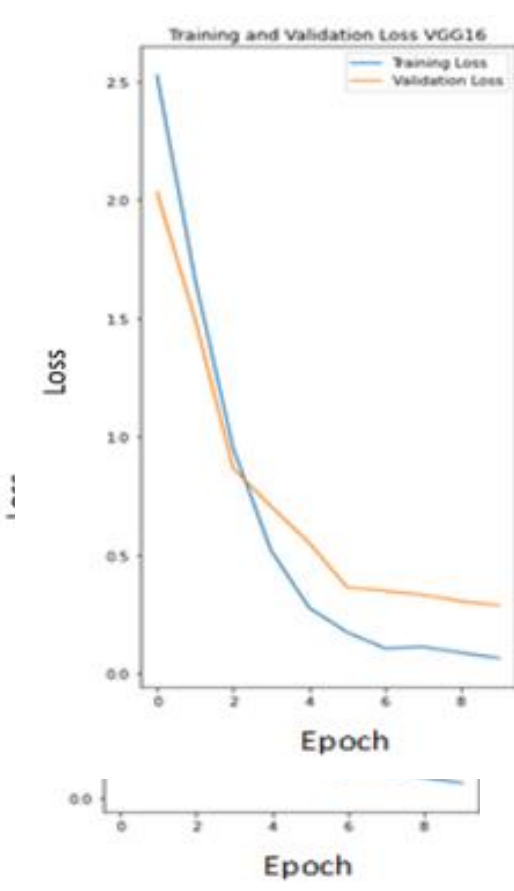

(a)

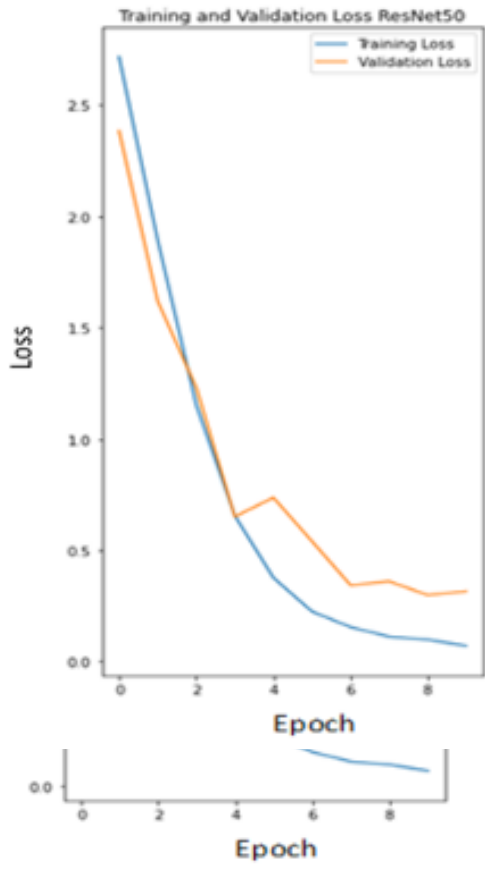

(b)

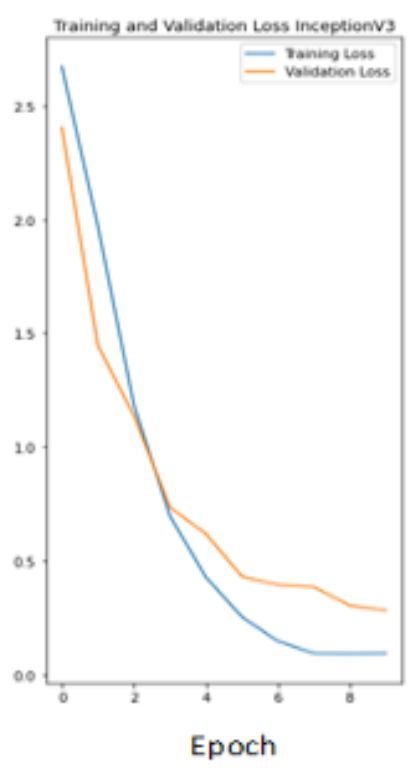

(c)

Figure 9. Training and validation loss produced by (a) VGG16, (b) ResNet50, and (c) Inception V3.
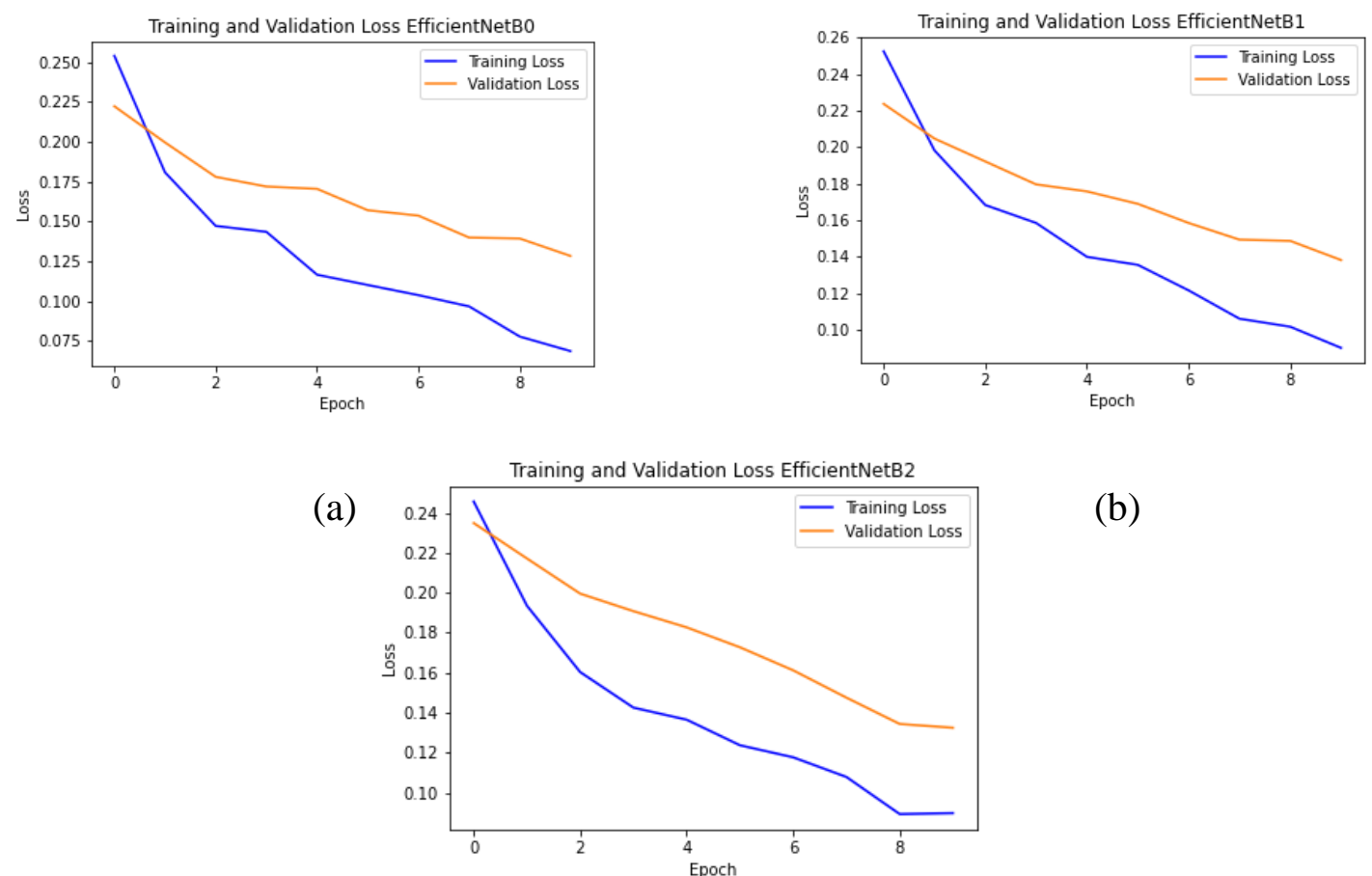

(b)

(c)

Figure 10. Training and validation loss produced by (a) EfficientNet B0, (b) EfficientNet B1, and (c) EfficientNet B2. 
It is shown in Figure 7 that VGG16, ResNet50, and Inception V3 algorithms have produced approximately similar percentages of accuracy, which range from $91 \%$ to $92 \%$, where ResNet50 is shown to outperform the rest of the algorithms by achieving the highest accuracy. Nevertheless, in terms of stability, VGG16 is shown to outperform the rest. Meanwhile, in Figure 8, EfficientNet algorithms have shown lower accuracy than VGG16, ResNet50, and Inception V3, where the accuracy produced by EfficientNetB0, EfficientNetB1, and EfficientNetB2 are only 63\%, 59\%, and 62\%, respectively. The validation losses produced by the VGG16, ResNet50, and InceptionV3 algorithms, as shown in Figure 9, are much lower than the validation losses produced by EfficientNetB0, EfficientNetB1, and EfficientNetB2 algorithms, as shown in Figure 10. This shows that the dataset used in the experiment is very complex and challenging for efficient classifiers.

Moreover, EfficientNet algorithms need to be trained by an extensive dataset such as the ImageNet dataset to efficiently classify the data, as Tan (2019) discussed. The effect of image size on the performance of the deep learning algorithms is also studied. For this purpose, only VGG16, ResNet50, and Inception V3 were considered as these three algorithms can produce high accuracy when classifying images from the chili plant growth dataset. The results are shown in Table 3, and it is observed that a larger image size slightly influenced the performance of VGG16 and Inception V3. Nevertheless, the accuracy of ResNet50 is greatly affected by the size of the image, where it is shown in Table 3 that largesized images decrease the accuracy of classification.

Table 3. Comparison of accuracy and loss for different image sizes.

\begin{tabular}{llllc}
\hline Algorithm & \multicolumn{3}{c}{$128 \times 128$ pixels } & $256 \times 256$ pixels \\
\cline { 2 - 5 } & Accuracy & Loss & Accuracy & Loss \\
\hline VGG16 & $91.60 \%$ & 0.2877 & $91.19 \%$ & 0.2505 \\
ResNet50 & $92.01 \%$ & 0.3162 & $87.91 \%$ & 0.4192 \\
InceptionV3 & $91.39 \%$ & 0.2828 & $90.57 \%$ & 0.3367 \\
\hline
\end{tabular}

Although VGG16, ResNet50, and InceptionV3 algorithms have shown good performance when classifying the growth stage of chili plants from images under a runaway condition, the performance of these algorithms when classifying images with different complexity is investigated as well. The training and validation accuracy produced by each algorithm is illustrated in Figure 11. The results showed that VGG16, ResNet50, and InceptionV3 produced inadequate training and validation accuracies of less than $80 \%$ and $30 \%$, respectively. Moreover, the algorithms showed inconsistent stability as the number of 
epoch increased. Hence, we can conclude that images under extreme exposure with a complex background consisting of multiple chili plants are too challenging for the classifiers.

Furthermore, the total number of images used to train and validate the algorithms is too small. This is due to the difficulty and challenges faced by the researchers to collect the data during the movement control order (MCO) period in Malaysia. Even so, the complex chili plant images will create more opportunities for the researchers to produce a highly accurate algorithm.

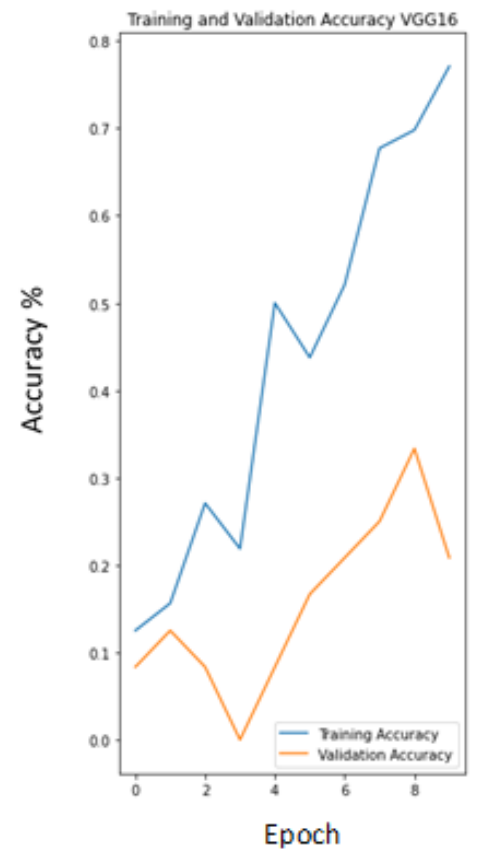

(a)

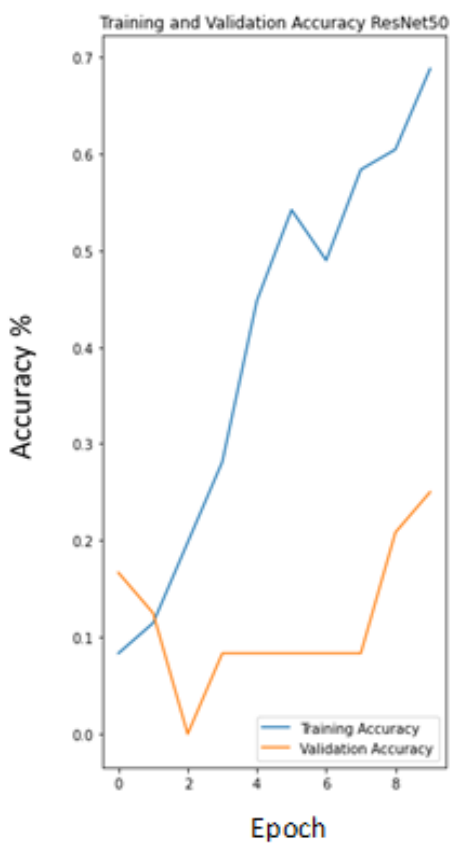

(b)

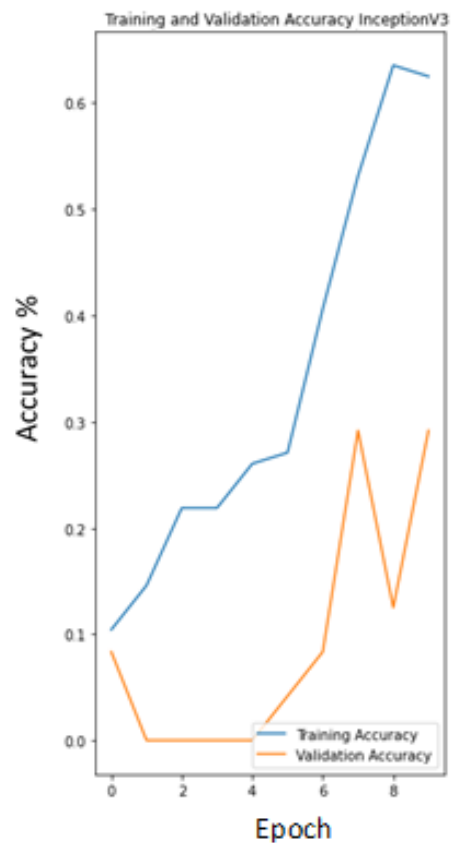

(c)

Figure 11. Training and validation accuracies produced by (a) VGG16, (b) ResNet50, and (c) Inception V3.

\section{Conclusions}

The efficacy of deep learning algorithms, namely VGG16, ResNet50, Inception V3, EfficientNet B0, EfficientNet B1and EfficientNet B2 algorithms in classifying the growth stage of chili plants from a dataset that consists of 2,320 plant images in a runaway condition is demonstrated. The experiment results showed that VGG16, ResNet50, and Inception V3 are far more accurate and stable than the EfficientNet algorithms. It is also found that the accuracy of the deep learning algorithms is greatly affected by the complexity of the images. Under extreme exposure, the algorithms have difficulty classifying the images correctly. The size of the dataset also plays a vital role in producing a highly accurate classifier. 
In conclusion, the VGG16, ResNet50, and Inception V3 algorithms have shown great potential for classifying the growth stage of chili plants. The performance of the algorithms can be further improved by exposing them to images with high complexity, such as images rotated at various angles, images scaled to various sizes and exposed to various lighting effects. This will create more opportunities for developing an advanced classifier.

Funding: This work was funded by the Ministry of Higher Education with grant number 5540078 (FRGS/1/2018/WAB01/UPM/02/30).

Acknowledgments: The authors would also want to thank Mrs. Noor Azlina Abdul Aziz for her support in this study.

Conflicts of Interest: The authors declare no conflict of interest.

\section{References}

Alhnaity, B., Pearson, S., Leontidis, G., et al. (2020). Using deep learning to predict plant growth and yield in greenhouse environments. Acta Horticulturae, (1296), 425-432. doi:https://doi.org/10.17660/ actahortic. 2020.1296 .55

Anugraheni, N. A., Suhendi, A., \& Bethanigtyas, H. (2019). Image processing of IoT based cherry tomato growth monitoring system. In 2019 6th International Conference on Instrumentation, Control, and Automation (ICA), 2019. pp. 207-210. doi:https://doi.org/10.1109/ICA.2019.8916680

Ayan, E., Erbay, H., \& Varçın, F. (2020). Crop pest classification with a genetic algorithm-based weighted ensemble of deep convolutional neural networks. Computers and Electronics in Agriculture, 179, 105809. doi: https://doi.org/10.1016/j.compag.2020.105809

Bahtiar, A. R., Santoso, A. J., \& Juhariah, J. (2020). Deep learning detected nutrient deficiency in chili. In 2020 8th International Conference on Information and Communication Technology (ICoICT), 2020. pp. 14. doi: https://doi.org/10.1109/ICoICT49345.2020.9166224

Bouman, C., \& Shapiro, M. (1994). A multiscale random field model for Bayesian image segmentation. IEEE Transactions On Image Processing, 3(2), 162-177. doi:https://doi.org/10.1109/83.277898

Chouhan, S. S., Kaul, A., \& Singh, U. P. (2019). A deep learning approach for the classification of diseased plant leaf images. In 2019 International Conference on Communication and Electronics Systems (ICCES), 2019, pp. 1168-1172. doi:https://doi.org/10.1109/ICCES45898.2019.9002201

Department of Statistics Malaysia. (2020). Department of Statistics Press Release Supply and Utilization Accounts Selected Agricultural Commodities, December, pp. 2013-2015.

Ferentinos, K. (2018). Deep learning models for plant disease detection and diagnosis. Computers And Electronics In Agriculture, 145, 311-318. doi:https://doi.org/10.1016/j.compag.2018.01.009 
Fuentes, A., Yoon, S., \& Park, D. (2019). Deep learning-based phenotyping system with glocal description of plant anomalies and symptoms. Frontiers In Plant Science, 10. doi:https://doi.org/10.3389/fpls.2019.01321

Guo, Y., Liu, Y., Oerlemans, A., Lao, S., Wu, S., \& Lew, M. (2016). Deep learning for visual understanding: A review. Neurocomputing, 187, 27-48. doi:https://doi.org/10.1016/j.neucom.2015.09.116

Habiba, S. U., Islam, M. K., \& Ahsan, S. M. M. (2019). Bangladeshi plant recognition using deep learning based leaf classification. In 2019 International Conference on Computer, Communication, Chemical, Materials and Electronic Engineering (IC4ME2), 2019. pp. 1-4. doi:https://doi.org/10.1109/IC4ME247184.2019.9036515

Haifa Group (2021). Crop Guide: Growing Peppers. https://www.haifa-group.com/articles/crop-guidegrowing-peppers.

Hao, X., Jia, J., Mateen Khattak, A., et al. (2020). Growing period classification of Gynura bicolor DC using GL-CNN. Computers And Electronics In Agriculture, 174, 105497. doi:https://doi.org/10.1016/j.compag.2020.105497

He, K., Zhang, X., Ren, S., et al. (2016). Deep residual learning for image recognition. In 2016 IEEE Conference on Computer Vision and Pattern Recognition (CVPR), 2016. pp. 770-778. doi:https://doi.org/10.1109/CVPR.2016.90

Keller, K., Kirchgeßner, N., Khanna, R., et al. (2018). Soybean leaf coverage estimation with machine learning and thresholding algorithms for field phenotyping. In 29th British Machine Vision Conference (BMVC), Newcastle upon Tyne, United Kingdom, 2018. p.32.

Khanramaki, M., Askari Asli-Ardeh, E., \& Kozegar, E. (2021). Citrus pests classification using an ensemble of deep learning models. Computers And Electronics In Agriculture, 186, 106192. doi:https://doi.org/10.1016/j.compag.2021.106192

Kim, M., Choi, E., Baek, G., et al. (2013). Lettuce growth prediction in plant factory using image processing technology. IFAC Proceedings Volumes, 46(4), 156-159. doi:https://doi.org/10.3182/20130327-3-jp3017.00036 .

Kingma, Diederik \& Ba, Jimmy. (2014). Adam: A method for stochastic optimization. In International Conference on Learning Representations, 2015. https://arxiv.org/abs/1412.6980.

Learning, M., \& Rai, H. (2021). Machine Learning vs Deep Learning. Data Science Stack Exchange. https://datascience.stackexchange.com/questions/16422/machine-learning-vs-deep-learning.

Li, C., Adhikari, R., Yao, Y., et al. (2020). Measuring plant growth characteristics using smartphone based image analysis technique in controlled environment agriculture. Computers And Electronics In Agriculture, 168, 105123. doi:https://doi.org/10.1016/j.compag.2019.105123

Li, X., Zeng, R., \& Liao, H. (2015). Improving crop nutrient efficiency through root architecture modifications. Journal Of Integrative Plant Biology, 58(3), 193-202. doi:https://doi.org/10.1111/jipb.12434 
Mochida, K., Koda, S., Inoue, K., et al. (2018). Computer vision-based phenotyping for improvement of plant productivity: A machine learning perspective. Gigascience, $8(1)$. doi:https://doi.org/10.1093/gigascience/giy153

Majlis Ilmu, Kerajaan Negara Brunei Darussalam (2018). Life Cycle of Plants. https://www.majlisilmu.gov.bn/Shared\%20Documents/Pameran/2018/WEEK\%205\%20Life_Cycle.p df

Norfadzilah, A. (2018). Screening of selected cucumber mosaic virus resistance chilli genotypes obtained from world vegetable center for high yield in Malaysian condition. In Transactions of the Malaysian Society of Plant Physiology, 25.

Namin, S.T., Esmaeilzadeh, M., Najafi, M., et al. (2018). Deep phenotyping: deep learning for temporal phenotype/genotype classification. Plant Methods, 14(1). doi:https://10.1186/s13007-018-0333-4

Othman, A. A. \& Tizhoosh, H. R (2010). Image thresholding using neural network. In 2010 10th International Conference on Intelligent Systems Design and Applications, 2010. pp. 1159-1164. doi:https://doi.org/10.1109/ISDA.2010.5687030

Otsu, N. (1979). A threshold selection method from gray-level histograms. IEEE Transactions On Systems, Man, And Cybernetics, 9(1), 62-66. doi:https://doi.org/10.1109/tsmc.1979.4310076

Peng, J., Kang, S., Ning, Z., et al. (2019). Residual convolutional neural network for predicting response of transarterial chemoembolization in hepatocellular carcinoma from CT imaging. European Radiology, 30(1), 413-424. doi:https://doi.org/10.1007/s00330-019-06318-1

Rangarajan, A.K., \& Purushothaman, R. (2020). Disease classification in eggplant using pre-trained VGG16 and MSVM. Scientific Reports, 10(1). doi:https://doi.org/10.1038/s41598-020-59108-x

Sakurai, S., Uchiyama, H., Shimada, A., et al.(2019). Plant growth prediction using convolutional LSTM. In VISIGRAPP 2019 - Proceedings of the 14th International Joint Conference on Computer Vision, Imaging and Computer Graphics Theory and Applications, Vol. 5. SciTePress.

Sandler, M., Howard, A., Zhu, M., et al. (2018). MobileNetV2: inverted residuals and linear bottlenecks. In 2018 IEEE/CVF Conference on Computer Vision and Pattern Recognition, 2018. pp. 4510-4520. doi:https://doi.org/10.1109/CVPR.2018.00474

Sharma, R. \& Kumar, R. (2017). Growth, flowering and yield of chilli, Capsicum annuum L. as influenced by spacing and growing conditions. International Journal Of Pure \& Applied Bioscience, 5(5), 524-527. doi:https://doi.org/10.18782/2320-7051.5858

Simonyan, K., \& Zisserman, A. (2021). Very Deep Convolutional Networks for Large-Scale Image Recognition. https://arxiv.org/abs/1409.1556v4.

Soethe, C., Steffens, C., Mattos, L., et al. (2016). Postharvest quality and functional compounds in "dedo-demoça" 'BRS Mari' pepper fruit at different stages of maturity. Ciência Rural, 46(8), 1322-1328. doi: https://doi.org/10.1590/0103-8478cr20141795 
Spalding, E., \& Miller, N. (2013). Image analysis is driving a renaissance in growth measurement. Current Opinion In Plant Biology, 16(1), 100-104. doi:https://doi.org/10.1016/j.pbi.2013.01.001

Subetha, T., Khilar, R., \& Subaja Christo, M. (2021). A comparative analysis on plant pathology classification using deep learning architecture - Resnet and VGG19. Materials Today: Proceedings. doi:https://doi.org/10.1016/j.matpr.2020.11.993

Sudianto, Herdiyeni, Y., Haristu, A., \& Hardhienata, M. (2020). Chilli quality classification using deep learning. In 2020 International Conference on Computer Science and Its Application in Agriculture (ICOSICA), 2020. pp. 1-5. doi: https://doi.org/10.1109/icosica49951.2020.9243176

Suhaimi, M.Y., Arshad, A.M., Hani, M., et al. (2016). Potential and viability of chilli cultivation using fertigation technology in Malaysia. International Journal of Innovation and Applied Studies, 17, 11141119.

Sujatha, R., Chatterjee, J., Jhanjhi, N., et al. (2021). Performance of deep learning vs machine learning in plant leaf disease detection. Microprocessors And Microsystems, 80, 103615. doi:https://doi.org/10.1016/j.micpro.2020.103615

Suroso, I., Soelistiadji, K., \& Pitoyo, J. (2016). Development of chilli growth control system using artificial neural network. IFAC Proceedings Volumes, 34(11), 234-237. doi:https://doi.org/10.1016/s1474$6670(17) 34139-3$

Szegedy, C., Liu, W., Jia, Y., et al. (2015). Going deeper with convolutions. In 2015 IEEE Conference on Computer Vision and Pattern Recognition (CVPR), 2015. pp. 1-9. doi:https://doi.org/10.1109/CVPR.2015.7298594.

Tan, M. (2019). EfficientNet: Improving accuracy and efficiency through AutoML and model scaling. Google AI Blog. https://ai.googleblog.com/2019/05/efficientnet-improving-accuracy-and.html.

Tan, M., \& Le, Q. (2019). EfficientNet: Rethinking model scaling for convolutional neural networks. arXiv.org. https://arxiv.org/abs/1905.11946v5.

Van Dijk, A., Kootstra, G., Kruijer, W., et al. (2021). Machine learning in plant science and plant breeding. Iscience, 24(1), 101890. doi:https://doi.org/10.1016/j.isci.2020.101890

Wang, X., Xuan, H., Evers, B., et al. (2019). High-throughput phenotyping with deep learning gives insight into the genetic architecture of flowering time in wheat. GigaScience, 8(11). doi:https://doi.org/10.1093/gigascience/giz120

Wayne. (2021). Malaysia Hydroponics Suregrow powder fertiliser (10L/50L/100L) for chilli (Special Mixture). https://malaysiahydroponics.com/malaysia-hydroponics-powder-chilli.html

Yasrab, R., Zhang, J., Smyth, P., et al. (2021). Predicting plant growth from time-series data using deep learning. Remote Sensing, 13(3), 331. doi:https://doi.org/10.3390/rs13030331 
Yusuf, N., Mohd Fazi, S., Ali, N., et al. (2016). Effects of Colletotrichum capsici infection on the growth and antioxidative response on defense mechanisms of Capsicum annuum. Malaysian Journal of Microbiology, 12(6), 455-462.

Yu, Z., Wong, H., \& Wen, G. (2011). A modified support vector machine and its application to image segmentation. Image and Vision Computing, 29(1), 29-40. doi:https://doi.org/10.1016/j.imavis.2010.08.003

Zhang, X., Zou, J., He, K., et al. (2016). Accelerating very deep convolutional networks for classification and detection. IEEE Transactions on Pattern Analysis And Machine Intelligence, 38(10), 1943-1955. doi:https://doi.org/10.1109/tpami.2015.2502579

Copyright $(\odot) 2021$ by Rozilan, D. M. M., et al. and HH Publisher. This work is licensed under the Creative Commons Attribution-NonCommercial 4.0 International Lisence (CC-BY-NC4.0) 